\title{
LETTER
}

\section{Perioperative hemodynamic therapy: quality improvement programs should help to resolve our uncertainty}

\author{
Frederic Michard*1, Maxime Cannesson ${ }^{2}$ and Benoit Vallet ${ }^{3}$ \\ See related commentary by MacDonald and Pearse, http://ccforum.com/content/15/3/122
}

We read with interest the commentary by MacDonald and Pearse [1] stating that only large randomized clinical trials (RCTs) can resolve our uncertainty regarding the value of perioperative hemodynamic therapy. Over the past 20 years, more than 20 small to medium size RCTs and several meta-analyses have shown that perioperative hemodynamic optimization improves post-operative outcome [2].

Despite this level of evidence, we fully agree with MacDonald and Pearse that uncertainty remains for many anesthetists [3]. This uncertainty is nicely illustrated by a recent survey showing that the vast majority of anesthetists do not follow perioperative hemodynamic protocols during high-risk surgery [4].

We are not convinced, however, that only large RCTs can resolve our uncertainty. First, a blind design is not applicable in this context. Second, manpower is rarely an issue when conducting a clinical trial with dedicated investigators while it may become a limitation when implementing a new treatment protocol in daily practice. Last but not least, the Hawthorne effect - a form of reactivity whereby caregivers improve or modify their behavior when participating in a study - may affect the outcome in both groups, rendering hazardous the extrapolation of RCT results to the real world. This is particularly true for perioperative hemodynamic therapy since the mere eyeballing of the arterial pressure waveform gives valuable information regarding fluid responsiveness. As a result, large and visible respiratory swings in arterial pressure will likely trigger fluid administration in patients who do not belong to the goaldirected therapy group.

Quality improvement research programs have emerged over the past 5 years and allow the evaluation of new

\footnotetext{
${ }^{*}$ Correspondence: michard.frederic@free.fr

${ }^{1}$ Critical Care, Edwards Lifesciences, Irvine, CA

Full list of author information is available at the end of the article
}

clinical strategies in real life conditions [5]. These beforeand-after evaluations could be applied to perioperative hemodynamic therapy and we believe they have strong potential to resolve our uncertainty. RCTs, definitely gold standards for double-blind drug trials, may not be the most appropriate method to assess the value of new monitoring strategies and treatment protocols in the real world.

\section{Abbreviations}

$\mathrm{RCT}$, randomized clinical trial.

\section{Competing interests}

FM is a Vice-President, Global Medical Strategy, at Edwards Lifesciences. The above statements do not support the use of any specific medical device. The other authors declare that they have no competing interests.

\section{Author details}

${ }^{1}$ Critical Care, Edwards Lifesciences, Irvine, CA. ${ }^{2}$ Department of Anesthesia and Perioperative Care, University of California Irvine, USA. ${ }^{3}$ Department of Anesthesia, University Hospital of Lille, France.

Published: 10 October 2011

\section{References}

1. McDonald N, Pearse RM: Peri-operative hemodynamic therapy: only large clinical trials can resolve our uncertainty. Crit Care 2011, 15:122.

2. Hamilton MA, Cecconi M, Rhodes A: A systematic review and meta-analysis on the use of preemptive hemodynamic intervention to improve postoperative outcomes in moderate and high-risk surgical patients. Anesth Analg 2011, 112:1392-1402.

3. Miller TE, Roche AM, Gan TJ: Poor adoption of hemodynamic optimization during major surgery: are we practicing substandard care? Anesth Analg 2011, 112:1274-1276.

4. Cannesson M, Pestel G, Ricks C, Hoeft A, Perel A: Hemodynamic monitoring and management in patients undergoing high risk surgery: a survey among American society of anesthesiologists and European society of anaesthesiology members. Crit Care 2011, 15:in press.

5. Pronovost P, Needham D, Berenholtz S, Sinopoli D, Chu H, Cosgrove S, Sexton B, Hyzy R, Welsh R, Roth G, Bander J, Kepros J, Goeschel C: An intervention to decrease catheter-related bloodstream infections in the ICU. N Engl J Med 2006, 355:2725-2732

doi:10.1186/cc10336

Cite this article as: Michard F, et al:: Perioperative hemodynamic therapy: quality improvement programs should help to resolve our uncertainty. Critical Care 2011, 15:445. 